\title{
Sample size for estimating the population of stink bugs in soybean crops $^{1}$
}

\author{
Tamanho de amostra para a estimação da população de percevejos na cultura de soja
}

Glauber Renato Stürmer ${ }^{2}$, Alberto Cargnelutti Filho ${ }^{3 *}$, Jerson Vanderlei Carús Guedes ${ }^{4}$ e Regis Felipe Stacke

\begin{abstract}
It is important to quantify the population of stink bugs in the soybean, in order to determine the actions which are necessary for their control to protect the crop from losses in production, together with a reduction in cost and less environmental impact. The objectives of this study were to determine the sample size (the number of sampling points) needed to estimate the average population density of the bugs, and to verify the variability in sample size for the phases and species of the bugs and the phenological stages of the plants. In an area of 6.16 ha of soybeans, a grid of 154 sampling points, spaced $20 \times 20 \mathrm{~m}$ apart, was laid out. Population-density data were collected for nymphs and adults of the species Dichelops furcatus (Fabricius, 1775), Piezodorus guildinii (Westwood, 1873), Edessa meditabunda (Fabricius, 1794), Nezara viridula (Linnaeus, 1758), Euschistus heros (Fabricius, 1794) and Chinavia sp. (Say, 1832) employing a vertical beat sheet at 14 different phenological stages. Measurements of central tendency and variability, the Morisita index and the k parameter of negative binomial distribution were all calculated. Homogeneity of variances was verified and the sample size calculated. There is variability in the sample size when estimating the average population density of bugs across the phases and species of bug and the phenological stages of the soybean. Smaller sample sizes are necessary for the nymphs of $P$. guildinii and the final phenological stages (R6, R7.1, R7.3 and R8.2). Thirty-six sampling sites are enough to estimate the average population density of bugs in the final phenological stages (R6, R7.1, R7 .3 and R8.2) at an error of estimation equal to $30 \%$ of the estimated mean and at a level of confidence of $95 \%$.
\end{abstract}

Key words: Sample sizing. Experimental error. Pentatomidae.

RESUMO - É importante quantificar a população de percevejos da soja, para determinar as ações de controle necessárias para proteger a lavoura das perdas na produção, com redução dos custos e menor impacto ambiental. Os objetivos deste trabalho foram determinar o tamanho de amostra (número de pontos amostrais) para a estimação da média de densidade populacional de percevejos e verificar a variabilidade do tamanho de amostra entre as fases e as espécies de percevejos e os estádios fenológicos. Em 6,16 ha de soja, foi demarcado um gride de 154 pontos amostrais, espaçados de $20 \times 20 \mathrm{~m}$. Foram coletados dados de densidade populacional de percevejos ninfas e adultos das espécies Dichelops furcatus (Fabricius, 1775), Piezodorus guildinii (Westwood, 1873), Edessa meditabunda (Fabricius, 1794), Nezara viridula (Linnaeus, 1758), Euschistus heros (Fabricius, 1794) e Chinavia sp. (Say, 1832), por meio do pano-de-batida vertical, em 14 estádios fenológicos. Foram calculadas medidas de tendência central e de variabilidade, índice de Morisita e parâmetro k da distribuição binomial negativa. Foi verificada a homogeneidade de variâncias e calculado o tamanho de amostra. Há variabilidade do tamanho de amostra para a estimação da média de densidade populacional de percevejos entre as fases e as espécies de percevejos e entre os estádios fenológicos da soja. Menores tamanhos de amostra são necessários para as ninfas de $P$. guildinii e os estádios fenológicos finais (R6, R7.1, R7.3 e R8.2). Trinta e seis pontos amostrais são suficientes para estimar a média de densidade populacional de percevejos, para um erro de estimação igual a 30\% da média estimada, com grau de confiança de $95 \%$, nos estádios fenológicos finais (R6, R7.1, R7.3 e R8.2).

Palavras-chave: Dimensionamento amostral. Erro experimental. Pentatomidae.

\footnotetext{
*Autor para correspondência

${ }^{1}$ Recebido para publicação em 07/09/2012; aprovado em 16/10/2013

Parte da Dissertação de Mestrado do primeiro autor apresentada ao Programa de Pós-graduação em Agronomia da Universidade Federal de Santa Maria. Auxílio financeiro da CAPES e CNPq

${ }^{2}$ Programa de Pós-Graduação em Agronomia/Universidade Federal de Santa Maria, Campus Universitário, Camobi, Santa Maria-RS, Brasil, 97.105-900,glauber.sturmer@gmail.com

${ }^{3}$ Departamento de Fitotecnia, Centro de Ciências Rurais, Universidade Federal de Santa Maria, Campus Universitário, Camobi, Santa MariaRS, Brasil, 97.105-900, alberto.cargnelutti.filho@gmail.com

${ }^{4}$ Departamento de Defesa Fitossanitária, Centro de Ciências Rurais, Universidade Federal de Santa Maria, Campus Universitário, Camobi, Santa Maria-RS, Brasil, 97.105-900,jerson.guedes@gmail.com,regis_felip@ hotmail.com
} 


\section{INTRODUCTION}

The soybean is one of the most important agricultural products in Brazil, being grown on 25 million hectares, with a production of 66.3 million tons of grain for the harvest of 2011/2012 (COMPANHIA NACIONAL DE ABASTECIMENTO, 2012). Grainsucking stink bugs are serious insect pests in the cultivation of soybeans (CORRÊA-FERREIRA; AZEVEDO, 2002). They are widespread throughout plantations in Brazil, and by feeding on the grains, reduce their weight and quality (CORRÊA-FERREIRA, 2005). Losses in the soybean caused by these bugs, at a population density of one stink bug $\mathrm{m}^{-2}$, range from 49 to $125 \mathrm{~kg} \mathrm{ha}^{-1}$ (GUEDES et al., 2012). Pest management in the soybean is carried out based on population levels, which should be quantified separately for each plantation, either for seed or grain production. Counting the bugs collected by means of a vertical beat sheet is a suitable method of quantifying the population of stink bugs (STÜRMER et al., 2012).

Sampling should be performed at different points throughout the plantation. Insufficient sampling points may lead to errors in the estimation of the average population density of the bugs, resulting in poor decisions being made regarding control of the insect pests, with consequent losses in grain yield or increases in the cost of production (GUEDES et al., 2012). Sample size (the number of sampling points) is directly proportional to the variability of the data and the desired level of confidence for the estimate, and inversely proportional to the permitted error of estimation, which is stipulated by the researcher. It therefore becomes necessary to define the number of sampling points, in order to estimate the average population density of bugs with the desired accuracy.

Population surveys and sample sizing of stink bugs in the soybean (COSTA; LINK, 1980; CULLEN et al., 2000; GUEDES et al., 2006; GUEDES et al., 2012; LOURENÇÃO et al., 2002; SANTOS, 2008) and of white grubs in areas of native pasture and under cultivation (CARGNELUTTI FILHO et al., 2011; SILVA; COSTA, 1998) have been carried out. For quantifying the density of stink bugs in the soybean, the recommendation is for the collection of 6 samples for areas of up to 10 ha, 8 samples for areas of 11-30 ha and 10 samples for areas of 30 to 100 ha. For areas larger than 100 ha, it is recommended to subdivide the area into plots of 100 ha (EMPRESA BRASILEIRA DE PEQUISA AGROPECUÁRIA, 2010). However, this recommendation does not take into account the phases and species of the bugs nor the phenological stage of the soybean. Additionally, no information was found on the estimation error of the mean using these recommendations or on the sample size (number of sampling points) necessary to quantify the population density of stink bugs in the soybean crop.

The objectives of this study were to determine the sample size (number of sampling points) for estimating the average population density of stink bugs, and to check the variability of the sample size across the phases and species of bug, and the stages of soybean.

\section{MATERIAL AND METHODS}

An experiment was carried out on a soybean crop in the agricultural year of 2010/2011, in an area of 6.16 ha, located at $29^{\circ} 42$ ' $24^{\prime \prime}$ S, 53'48' $42^{\prime}$ ' W and an altitude of $95 \mathrm{~m}$. The soybean cultivar 'BMX Potência $R R$ ' was seeded on 29 October 2010, in rows spaced 0.5 $\mathrm{m}$ apart, at a density of 25 plants $\mathrm{m}^{-2}$. Control of weeds and diseases was conducted in accordance with cropresearch recommendations (RPS RS-2010). Insecticide for contolling the stink bugs was not used. A grid was marked out, of 154 sampling points, spaced $20 \times 20 \mathrm{~m}$ apart. Bugs were collected at each of the sample points, using the vertical beat-sheet method of sampling, for 14 phenological stages of the soybean (V7, V9, V11, R1, R2, R3, R4, R5.1, R5.3, R5.5, R6, R7.1, R7.3 and R8.2), defined according to the scale proposed by Ritchie et al. (1982).

The vertical beat sheet consisted of a wooden stick at the upper end and a $100 \mathrm{~mm}$ tube of chlorinated polyvinyl, cut in half lengthwise, at the lower end, connected by a white fabric of $1 \mathrm{~m}$ in length, with its height adjusted to that of the soybean plants. The tube served as a collector for the bugs (Figure 1). To collect the bugs, the cloth was placed vertically between the rows of crops, and the plants from just one row were shaken against the surface of the cloth. This procedure was carried out on two metres of the row of soybeans, in order to sample an area of $1 \mathrm{~m}^{2}$.

For each of the 2,156 collections (154 sampling points/phonological stage $\times 14$ phonological stages of the soybean) with an area of $1 \mathrm{~m}^{2}$, the number of stink bug nymphs and adults were counted for the species Dichelops furcatus (Fabricius, 1775), Piezodorus guildinii (Westwood, 1873), Edessa meditabunda (Fabricius, 1794), Nezara viridula (Linnaeus, 1758), Euschistus heros (Fabricius, 1794) and Chinavia sp. (Say, 1832), thereby obtaining 12 variables ( 2 phases $\times 6$ species). Three more variables were then obtained, i.e. the total nymphs, adults and nymphs + adults, for each phonological stage, regardless of species.

For these 15 variables, for each phenological stage and from the population density of bugs at the 154 
Figure 1 - Representation of the vertical beat sheet used for sampling bugs in the soybean crop

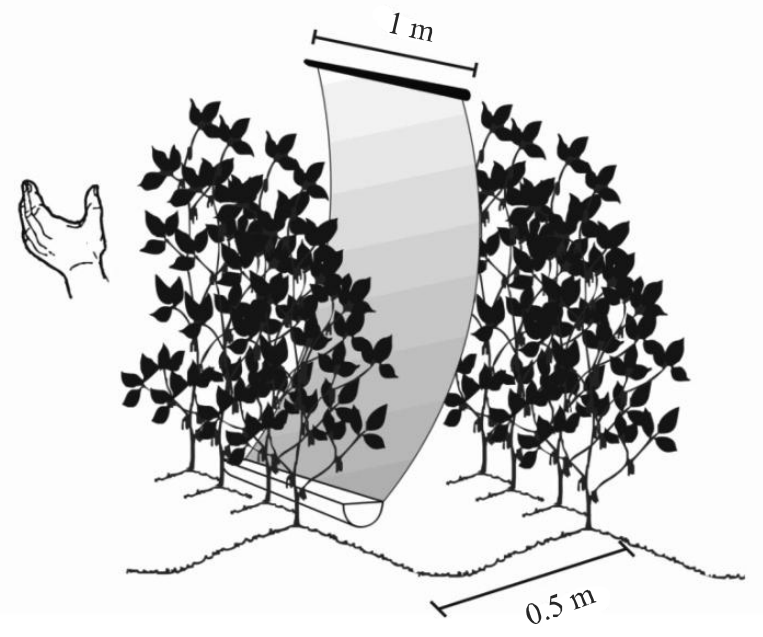

Source: Prepared by the authors

sampling points, the following statistics were calculated: minimum, maximum, mean (m), standard deviation (s), variance $\left(\mathrm{s}^{2}\right)$ and coefficient of variation. Next, the Morisita index $\left(\mathrm{I}_{\delta}\right)$ (MORISITA, 1962) and the k parameter of the negative binomial distribution were calculated employing expressions 1 and 2 respectively:

$$
I_{\delta}=\frac{n\left(\sum_{i=1}^{n} x_{i}^{2}-\sum_{i=1}^{n} x_{i}\right)}{\left(\sum_{i=1}^{n} x_{i}\right)^{2}-\sum_{i=1}^{n} x_{i}}
$$

$\mathrm{k}=\frac{\mathrm{m}^{2}}{\left(\mathrm{~s}^{2}-\mathrm{m}\right)}$

where $\mathrm{n}$ is the number of sample points $(\mathrm{n}=154), \mathrm{x}_{\mathrm{i}}$ is the number of bugs at the $\mathrm{i}^{\text {th }}$ sampling point, and $\mathrm{m}$ is the mean and $s^{2}$ the variance of the sample.

The F-test (one-sided) was then applied to the population-density data of the bugs in order to verify the homogeneity of the variances between the two phases of the bug within each combination of species and phenological stage, between the six species within each combination of phase and phenological stage and between the 14 phenological stages of the soybean within each combination of phase and species.

For each of the 15 variables at each phenological stage, taking as a basis the 154 sampling points $(n=154)$, the sample size was calculated (number of sampling points, $\eta$ ) for the semi-amplitudes of the confidence interval (errors of estimation) equal to $10,20,30,40$ and $50 \%$ (D) of the estimate of the mean $(\mathrm{m})$ of the population density of the bugs, expressed as bugs $\mathrm{m}^{-2}$, with a level of confidence $(1-\alpha)$ of $95 \%$, by means of the expression: $\eta=\left(t^{2}{ }_{\alpha / 2} S^{2}\right) /(D m)^{2}$ (BUSSAB; MORETTIN, 2004). In this expression, $t_{\alpha / 2}$ is the critical value of the Student's t-distribution, where the area on the right is equal to $\alpha / 2$, i.e. the value of $t$, such that $P\left(t>t_{\alpha / 2}\right)=\alpha / 2$, with (n-1) degrees of freedom, with $\alpha=5 \%$ probability of error and $s^{2}$ the estimation of variance. Then, making $\eta$ equal to 154 sampling points, the error of estimation was calculated as a percentage of the estimate of the mean (m) using expression 3:

$$
\mathrm{D}=\left(100 \mathrm{t}_{\alpha / 2} \mathrm{~s}\right) /(\sqrt{\eta \mathrm{m}})
$$

where $\mathrm{s}$ is the estimated standard deviation.

Statistical analyses were carried out employing the Microsoft Office Excel $®$ software with all decimal places being used in the intermediate calculations.

\section{RESULTS AND DISCUSSION}

There was variability in the population densities of the bugs for their phases and species and for the phonological stages of the soybeans (Tables 1, 2 and 3). Nymphs were seen in greater quantities than were adults. Piezodorus guildinii predominated over the other species of stink bug. Across the phenological stages, population density increased gradually from the initial stages (V7 and V9) to the final stages (R7.1, R7.3 and R8.2), with the peak population of bugs of $19.34 \mathrm{~m}^{-2}$ occurring for R7.3 (Table 3). These results agree with Silva et al. (2007) and Maziero et al. (2009), who showed that during the reproductive stage of the soybean, the stink-bug population may be made up of more than $70 \%$ nymphs compared to adults. A predominance of $P$. guildinii has been found in surveys of stink-bug populations in the soybean (LOURENÇÃO et al., 2002). Higher population densities of the bugs were verified by Belorte, Ramiro and Faria (2003) at stages R7 and R8.

The largest stink-bug infestation occurred during the reproductive stage of the soybean, this phase being the most sensitive to the pest. The bugs colonize during the vegetative phase, but the damage is caused when the bugs feed, starting from the R3 stage of the bean. The decrease in the bug population which occurred in the evaluation that took place in R8.2, can be explained by migration to areas of refuge and the crop no longer being a food preference. Close to the bean harvest, the bugs begin their dispersal to host plants and to diapause niches, in which they remain until the next crop (MEDEIROS; MEGIER, 2009).

In general, the coefficients of variation $(\mathrm{CV})$ of stink-bug population density were higher for the species D. furcatus, E. meditabunda, N. viridula, E. heros and 
Table 1 - Minimum, maximum, mean, standard deviation (s), variance $\left(\mathrm{s}^{2}\right)$, coefficient of variation $(\mathrm{CV} \%)$, Morisita index $\left(\mathrm{I}_{\delta}\right)$ and k parameter of the negative binomial distribution, of the population density for nymphs of the stink-bug species Dichelops furcatus, Piezodorus guildinii, Edessa meditabunda, Nezara viridula, Euschistus heros and Chinavia sp., expressed as stink bugs $\mathrm{m}^{-2}$, based on 154 points each of $1 \mathrm{~m}^{2}$ in area, for 14 phenological stages in the soybean

\begin{tabular}{|c|c|c|c|c|c|c|c|c|c|c|c|c|c|c|}
\hline \multirow{2}{*}{ Statistic } & \multicolumn{14}{|c|}{ Phenological stage ${ }^{(1)}$-. } \\
\hline & V7 & V9 & V11 & $\mathrm{R} 1$ & $\mathrm{R} 2$ & R3 & $\mathrm{R} 4$ & R5.1 & R5.3 & R5.5 & R6 & R7.1 & $\mathrm{R} 7.3$ & $\mathrm{R} 8.2$ \\
\hline \multicolumn{15}{|c|}{ Dichelops furcatus } \\
\hline Mínimum & 0.00 & 0.00 & 0.00 & 0.00 & 0.00 & 0.00 & 0.00 & 0.00 & 0.00 & 0.00 & 0.00 & 0.00 & 0.00 & 0.00 \\
\hline Maximum & 0.00 & 0.00 & 1.00 & 1.00 & 2.00 & 2.00 & 2.00 & 1.00 & 2.00 & 2.00 & 4.00 & 5.00 & 7.00 & 6.00 \\
\hline Mean & 0.00 & 0.00 & 0.01 & 0.04 & 0.09 & 0.13 & 0.08 & 0.04 & 0.07 & 0.07 & 0.37 & 0.80 & 1.06 & 0.42 \\
\hline $\mathrm{s}$ & 0.00 & 0.00 & 0.11 & 0.19 & 0.31 & 0.37 & 0.33 & 0.19 & 0.28 & 0.28 & 0.77 & 1.14 & 1.32 & 1.25 \\
\hline $\mathrm{s}^{2}$ & 0.00 & 0.00 & 0.01 & 0.04 & 0.10 & 0.14 & 0.11 & 0.04 & 0.08 & 0.08 & 0.59 & 1.30 & 1.73 & 1.55 \\
\hline $\mathrm{CV}(\%)$ & - & - & 874.62 & 498.28 & 341.28 & 287.99 & 428.59 & 498.28 & 395.56 & 395.56 & 207.10 & 142.70 & 123.66 & 295.22 \\
\hline $\mathrm{I}_{\delta}$ & - & - & $0.00^{\text {ns }}$ & $0.00^{\mathrm{ns}}$ & $1.69^{\mathrm{ns}}$ & $1.62^{\mathrm{ns}}$ & $7.00 *$ & $0.00^{\mathrm{ns}}$ & $2.80^{\mathrm{ns}}$ & $2.80^{\mathrm{ns}}$ & $2.61 *$ & $1.79 *$ & $1.59 *$ & $7.40^{*}$ \\
\hline $\mathrm{k}$ & - & - & -1.99 & -1.19 & 1.55 & 1.68 & 0.18 & -1.19 & 0.61 & 0.61 & 0.63 & 1.27 & 1.69 & 0.16 \\
\hline \multicolumn{15}{|c|}{ Piezodorus guildinii } \\
\hline Mínimum & 0.00 & 0.00 & 0.00 & 0.00 & 0.00 & 0.00 & 0.00 & 0.00 & 0.00 & 0.00 & 0.00 & 0.00 & 0.00 & 1.00 \\
\hline Maximum & 0.00 & 1.00 & 1.00 & 5.00 & 2.00 & 5.00 & 3.00 & 5.00 & 2.00 & 11.00 & 13.00 & 33.00 & 35.00 & 36.00 \\
\hline Mean & 0.00 & 0.02 & 0.01 & 0.08 & 0.08 & 0.22 & 0.19 & 0.26 & 0.11 & 0.32 & 2.26 & 7.11 & 10.31 & 8.01 \\
\hline $\mathrm{s}$ & 0.00 & 0.14 & 0.08 & 0.49 & 0.31 & 0.64 & 0.48 & 0.66 & 0.35 & 1.05 & 2.77 & 6.12 & 6.27 & 4.56 \\
\hline $\mathrm{s}^{2}$ & 0.00 & 0.02 & 0.01 & 0.24 & 0.10 & 0.41 & 0.23 & 0.44 & 0.12 & 1.11 & 7.67 & 37.47 & 39.35 & 20.76 \\
\hline $\mathrm{CV}(\%)$ & - & 711.77 & $1,240.97$ & 631.65 & 402.70 & 289.48 & 255.93 & 255.93 & 320.26 & 330.71 & 122.56 & 86.09 & 60.84 & 56.91 \\
\hline$I_{\delta}$ & - & $0.00^{\mathrm{ns}}$ & - & $30.33 *$ & $4.67 *$ & $4.94 *$ & $2.28^{*}$ & $3.75^{*}$ & $2.26^{\mathrm{ns}}$ & $8.90 *$ & $2.06^{*}$ & $1.60 *$ & $1.27 *$ & $1.20 *$ \\
\hline $\mathrm{k}$ & - & -1.49 & - & 0.04 & 0.30 & 0.26 & 0.81 & 0.37 & 0.83 & 0.13 & 0.94 & 1.67 & 3.66 & 5.02 \\
\hline \multicolumn{15}{|c|}{ Edessa meditabunda } \\
\hline Mínimum & 0.00 & 0.00 & 0.00 & 0.00 & 0.00 & 0.00 & 0.00 & 0.00 & 0.00 & 0.00 & 0.00 & 0.00 & 0.00 & 0.00 \\
\hline Maximum & 0.00 & 0.00 & 0.00 & 0.00 & 0.00 & 3.00 & 4.00 & 2.00 & 1.00 & 2.00 & 5.00 & 5.00 & 13.00 & 4.00 \\
\hline Mean & 0.00 & 0.00 & 0.00 & 0.00 & 0.00 & 0.04 & 0.11 & 0.11 & 0.05 & 0.16 & 0.15 & 0.24 & 0.41 & 0.22 \\
\hline $\mathrm{s}$ & 0.00 & 0.00 & 0.00 & 0.00 & 0.00 & 0.28 & 0.51 & 0.39 & 0.21 & 0.40 & 0.56 & 0.71 & 1.25 & 0.60 \\
\hline $\mathrm{s}^{2}$ & 0.00 & 0.00 & 0.00 & 0.00 & 0.00 & 0.08 & 0.26 & 0.15 & 0.04 & 0.16 & 0.31 & 0.51 & 1.55 & 0.36 \\
\hline $\mathrm{CV}(\%)$ & - & - & - & - & - & 711.77 & 458.08 & 352.17 & 459.75 & 255.51 & 373.33 & 297.39 & 304.38 & 270.31 \\
\hline $\mathrm{I}_{\delta}$ & - & - & - & - & - & $30.80 *$ & $13.59^{*}$ & $4.53^{*}$ & $0.00^{\mathrm{ns}}$ & $1.12^{\mathrm{ns}}$ & $8.52 *$ & $5.78^{*}$ & $7.89^{*}$ & $3.84^{*}$ \\
\hline $\mathrm{k}$ & - & - & - & - & - & 0.04 & 0.08 & 0.30 & -1.16 & 8.94 & 0.14 & 0.21 & 0.15 & 0.36 \\
\hline \multicolumn{15}{|c|}{ Nezara viridula } \\
\hline Mínimum & 0.00 & 0.00 & 0.00 & 0.00 & 0.00 & 0.00 & 0.00 & 0.00 & 0.00 & 0.00 & 0.00 & 0.00 & 0.00 & 0.00 \\
\hline Maximum & 0.00 & 0.00 & 4.00 & 0.00 & 1.00 & 1.00 & 2.00 & 3.00 & 1.00 & 3.00 & 3.00 & 16.00 & 15.00 & 7.00 \\
\hline Mean & 0.00 & 0.00 & 0.03 & 0.00 & 0.07 & 0.06 & 0.04 & 0.09 & 0.05 & 0.12 & 0.14 & 0.50 & 0.55 & 0.31 \\
\hline $\mathrm{s}$ & 0.00 & 0.00 & 0.32 & 0.00 & 0.26 & 0.25 & 0.23 & 0.39 & 0.21 & 0.41 & 0.46 & 1.93 & 1.80 & 0.89 \\
\hline $\mathrm{s}^{2}$ & 0.00 & 0.00 & 0.10 & 0.00 & 0.07 & 0.06 & 0.05 & 0.15 & 0.04 & 0.17 & 0.21 & 3.73 & 3.23 & 0.79 \\
\hline $\mathrm{CV}(\%)$ & - & - & $1,240.97$ & - & 361.73 & 380.71 & 578.27 & 423.96 & 459.75 & 351.98 & 324.39 & 386.20 & 325.58 & 290.97 \\
\hline $\mathrm{I}_{\delta}$ & - & - & $154.00^{*}$ & - & $0.00^{\mathrm{ns}}$ & $0.00^{\mathrm{ns}}$ & $10.27 *$ & $8.46^{*}$ & $0.00^{\mathrm{ns}}$ & $5.03 *$ & $4.67 *$ & $14.00 *$ & $9.84 *$ & $6.27 *$ \\
\hline $\mathrm{k}$ & - & - & 0.01 & - & -1.09 & -1.10 & 0.13 & 0.14 & -1.16 & 0.26 & 0.28 & 0.08 & 0.11 & 0.19 \\
\hline \multicolumn{15}{|c|}{ Euschistus heros } \\
\hline Mínimum & 0.00 & 0.00 & 0.00 & 0.00 & 0.00 & 0.00 & 0.00 & 0.00 & 0.00 & 0.00 & 0.00 & 0.00 & 0.00 & 0.00 \\
\hline Maximum & 0.00 & 0.00 & 0.00 & 0.00 & 1.00 & 0.00 & 1.00 & 2.00 & 1.00 & 2.00 & 4.00 & 3.00 & 1.00 & 4.00 \\
\hline Mean & 0.00 & 0.00 & 0.00 & 0.00 & 0.01 & 0.00 & 0.01 & 0.03 & 0.01 & 0.01 & 0.19 & 0.07 & 0.05 & 0.08 \\
\hline $\mathrm{s}$ & 0.00 & 0.00 & 0.00 & 0.00 & 0.08 & 0.00 & 0.11 & 0.20 & 0.11 & 0.16 & 0.67 & 0.35 & 0.21 & 0.43 \\
\hline $\mathrm{s}^{2}$ & 0.00 & 0.00 & 0.00 & 0.00 & 0.01 & 0.00 & 0.01 & 0.04 & 0.01 & 0.03 & 0.45 & 0.12 & 0.04 & 0.18 \\
\hline
\end{tabular}


Continuation of Table 1

\begin{tabular}{|c|c|c|c|c|c|c|c|c|c|c|c|c|c|c|}
\hline $\mathrm{CV}(\%)$ & - & - & - & - & $1,240.97$ & - & 874.62 & 755.78 & 874.62 & $1,240.97$ & 342.61 & 483.05 & 459.75 & 505.89 \\
\hline $\mathrm{I}_{\delta}$ & - & - & - & - & - & - & $0.00^{\mathrm{ns}}$ & $25.67 *$ & $0.00^{\mathrm{ns}}$ & $154.00^{*}$ & $7.79 *$ & $11.20 *$ & $0.00^{\mathrm{ns}}$ & $15.79 *$ \\
\hline $\mathrm{k}$ & - & - & - & - & - & - & -1.99 & 0.05 & -1.99 & 0.01 & 0.15 & 0.11 & -1.16 & 0.07 \\
\hline \multicolumn{15}{|c|}{ Chinavia sp. } \\
\hline Mínimum & 0.00 & 0.00 & 0.00 & 0.00 & 0.00 & 0.00 & 0.00 & 0.00 & 0.00 & 0.00 & 0.00 & 0.00 & 0.00 & 0.00 \\
\hline Maximum & 0.00 & 0.00 & 0.00 & 0.00 & 2.00 & 2.00 & 2.00 & 3.00 & 5.00 & 38.00 & 28.00 & 14.00 & 43.00 & 9.00 \\
\hline Mean & 0.00 & 0.00 & 0.00 & 0.00 & 0.02 & 0.07 & 0.10 & 0.17 & 0.18 & 0.52 & 0.80 & 1.06 & 2.08 & 1.01 \\
\hline s & 0.00 & 0.00 & 0.00 & 0.00 & 0.18 & 0.28 & 0.39 & 0.47 & 0.58 & 3.11 & 3.13 & 2.10 & 4.44 & 1.69 \\
\hline $\mathrm{s}^{2}$ & 0.00 & 0.00 & 0.00 & 0.00 & 0.03 & 0.08 & 0.15 & 0.22 & 0.33 & 9.70 & 9.80 & 4.41 & 19.72 & 2.86 \\
\hline $\mathrm{CV}(\%)$ & - & - & - & - & 922.54 & 395.56 & 402.70 & 277.61 & 317.26 & 599.61 & 391.86 & 198.37 & 213.07 & 167.02 \\
\hline $\mathrm{I} \delta$ & - & - & - & - & $51.33 *$ & $2.80^{\mathrm{ns}}$ & $7.33^{*}$ & $2.84^{*}$ & $5.70^{*}$ & $35.23 *$ & $15.13^{*}$ & $3.99 *$ & $5.05^{*}$ & $2.80 *$ \\
\hline $\mathrm{k}$ & - & - & - & - & 0.03 & 0.61 & 0.17 & 0.56 & 0.22 & 0.03 & 0.07 & 0.33 & 0.25 & 0.55 \\
\hline
\end{tabular}

(1) Developmental stages of the soybean according to Ritchie et al. (1982), adapted by Yorinori (1996). * Morisita index different from 1 by the $\chi 2$ test at $5 \%$. ${ }^{\text {ns }}$ Non-significant. - Not calculated where no stink bugs, or only 1 , were found

Table 2 - Minimum, maximum, mean, standard deviation (s), variance $\left(\mathrm{s}^{2}\right)$, coefficient of variation $(\mathrm{CV} \%)$, Morisita index $\left(\mathrm{I}_{\delta}\right)$ and k parameter of the negative binomial distribution, of the population density for adults of the stink-bug species Dichelops furcatus, Piezodorus guildinii, Edessa meditabunda, Nezara viridula, Euschistus heros and Chinavia sp., expressed as stink bugs $\mathrm{m}^{-2}$, based on 154 points each of $1 \mathrm{~m}^{2}$ in area, for 14 phenological stages in the soybean

\begin{tabular}{|c|c|c|c|c|c|c|c|c|c|c|c|c|c|c|}
\hline \multirow{2}{*}{ Statistic } & \multicolumn{14}{|c|}{ - } \\
\hline & V7 & V9 & V11 & $\mathrm{R} 1$ & $\mathrm{R} 2$ & R3 & $\mathrm{R} 4$ & R5.1 & R5.3 & R5.5 & R6 & R7.1 & $\mathrm{R} 7.3$ & $\mathrm{R} 8.2$ \\
\hline & \multicolumn{14}{|c|}{ Dichelops furcatus } \\
\hline Mínimum & 0.00 & 0.00 & 0.00 & 0.00 & 0.00 & 0.00 & 0.00 & 0.00 & 0.00 & 0.00 & 0.00 & 0.00 & 0.00 & 0.00 \\
\hline Maximum & 1.00 & 2.00 & 2.00 & 1.00 & 3.00 & 2.00 & 3.00 & 2.00 & 4.00 & 3.00 & 2.00 & 3.00 & 5.00 & 5.00 \\
\hline Mean & 0.01 & 0.08 & 0.06 & 0.04 & 0.05 & 0.12 & 0.17 & 0.13 & 0.18 & 0.19 & 0.05 & 0.24 & 0.70 & 0.72 \\
\hline $\mathrm{s}$ & 0.08 & 0.29 & 0.30 & 0.19 & 0.29 & 0.35 & 0.47 & 0.37 & 0.49 & 0.50 & 0.25 & 0.56 & 1.11 & 1.13 \\
\hline$s^{2}$ & 0.01 & 0.09 & 0.09 & 0.04 & 0.08 & 0.12 & 0.22 & 0.14 & 0.24 & 0.25 & 0.06 & 0.31 & 1.23 & 1.29 \\
\hline $\mathrm{CV}(\%)$ & $1,240.97$ & 375.01 & 454.91 & 498.28 & 633.38 & 283.03 & 277.61 & 287.99 & 270.14 & 256.35 & 481.80 & 233.40 & 158.17 & 157.43 \\
\hline $\mathrm{I}_{\delta}$ & - & $2.33^{\mathrm{ns}}$ & $6.84^{*}$ & $0.00^{\mathrm{ns}}$ & $22.00 *$ & $0.90^{\mathrm{ns}}$ & $2.84^{*}$ & $1.62^{\mathrm{ns}}$ & $2.85^{*}$ & $2.48 *$ & $5.50 *$ & $2.31 *$ & $2.08 *$ & $2.09 *$ \\
\hline \multirow[t]{2}{*}{$\mathrm{k}$} & - & 0.81 & 0.19 & -1.19 & 0.06 & -10.55 & 0.56 & 1.68 & 0.56 & 0.70 & 0.25 & 0.78 & 0.93 & 0.92 \\
\hline & \multicolumn{14}{|c|}{ Piezodorus guildinii } \\
\hline Mínimum & 0.00 & 0.00 & 0.00 & 0.00 & 0.00 & 0.00 & 0.00 & 0.00 & 0.00 & 0.00 & 0.00 & 0.00 & 0.00 & 0.00 \\
\hline Maximum & 2.00 & 1.00 & 1.00 & 2.00 & 2.00 & 3.00 & 2.00 & 3.00 & 2.00 & 3.00 & 4.00 & 9.00 & 19.00 & 13.00 \\
\hline Mean & 0.02 & 0.02 & 0.01 & 0.06 & 0.13 & 0.15 & 0.10 & 0.19 & 0.22 & 0.29 & 0.31 & 1.08 & 3.59 & 3.80 \\
\hline $\mathrm{s}$ & 0.18 & 0.14 & 0.11 & 0.26 & 0.41 & 0.44 & 0.33 & 0.48 & 0.54 & 0.63 & 0.65 & 1.45 & 3.21 & 2.74 \\
\hline $\mathrm{s}^{2}$ & 0.03 & 0.02 & 0.01 & 0.07 & 0.17 & 0.19 & 0.11 & 0.23 & 0.29 & 0.39 & 0.43 & 2.11 & 10.33 & 7.53 \\
\hline $\mathrm{CV}(\%)$ & 922.54 & 711.77 & 874.62 & 447.70 & 313.75 & 294.33 & 314.52 & 255.93 & 244.26 & 214.04 & 209.18 & 134.81 & 89.53 & 72.26 \\
\hline$I_{\delta}$ & $51.33^{*}$ & $0.00^{\mathrm{ns}}$ & $0.00^{\mathrm{ns}}$ & $4.28^{\mathrm{ns}}$ & $3.24 *$ & $3.04^{*}$ & $1.28^{\mathrm{ns}}$ & $2.28 *$ & $2.47^{*}$ & $2.18^{*}$ & $2.18^{*}$ & $1.89^{*}$ & $1.52^{*}$ & $1.26^{*}$ \\
\hline \multirow[t]{2}{*}{$\mathrm{k}$} & 0.03 & -1.49 & -1.99 & 0.34 & 0.47 & 0.51 & 3.74 & 0.81 & 0.70 & 0.86 & 0.86 & 1.12 & 1.91 & 3.86 \\
\hline & \multicolumn{14}{|c|}{ Edessa meditabunda } \\
\hline Mínimum & 0.00 & 0.00 & 0.00 & 0.00 & 0.00 & 0.00 & 0.00 & 0.00 & 0.00 & 0.00 & 0.00 & 0.00 & 0.00 & 0.00 \\
\hline Maximum & 0.00 & 1.00 & 0.00 & 1.00 & 0.00 & 1.00 & 1.00 & 3.00 & 1.00 & 3.00 & 2.00 & 2.00 & 2.00 & 2.00 \\
\hline Mean & 0.00 & 0.01 & 0.00 & 0.01 & 0.00 & 0.02 & 0.03 & 0.07 & 0.07 & 0.08 & 0.14 & 0.11 & 0.18 & 0.19 \\
\hline $\mathrm{s}$ & 0.00 & 0.11 & 0.00 & 0.11 & 0.00 & 0.14 & 0.18 & 0.35 & 0.26 & 0.38 & 0.43 & 0.37 & 0.47 & 0.44 \\
\hline $\mathrm{s}^{2}$ & 0.00 & 0.01 & 0.00 & 0.01 & 0.00 & 0.02 & 0.03 & 0.12 & 0.07 & 0.14 & 0.19 & 0.14 & 0.22 & 0.19 \\
\hline $\mathrm{CV}(\%)$ & - & 874.62 & - & 874.62 & - & 711.77 & 547.67 & 483.05 & 361.73 & 448.21 & 304.00 & 336.60 & 269.93 & 233.33 \\
\hline $\mathrm{I}_{\delta}$ & - & $0.00^{\mathrm{ns}}$ & - & $0.00^{\mathrm{ns}}$ & - & $0.00^{\mathrm{ns}}$ & $0.00^{\text {ns }}$ & $11.20 *$ & $0.00^{\mathrm{ns}}$ & $9.87^{*}$ & $3.33^{*}$ & $3.40 *$ & $2.63 *$ & $1.14^{\mathrm{ns}}$ \\
\hline $\mathrm{k}$ & - & -1.99 & - & -1.99 & - & -1.49 & -1.24 & 0.11 & -1.09 & 0.12 & 0.45 & 0.44 & 0.63 & 7.46 \\
\hline
\end{tabular}


Continuation of Table 2

\begin{tabular}{|c|c|c|c|c|c|c|c|c|c|c|c|c|c|c|}
\hline & \multicolumn{14}{|c|}{ Nezara viridula } \\
\hline Mínimum & 0.00 & 0.00 & 0.00 & 0.00 & 0.00 & 0.00 & 0.00 & 0.00 & 0.00 & 0.00 & 0.00 & 0.00 & 0.00 & 0.00 \\
\hline Maximum & 1.00 & 1.00 & 1.00 & 1.00 & 1.00 & 3.00 & 2.00 & 1.00 & 1.00 & 1.00 & 3.00 & 3.00 & 6.00 & 2.00 \\
\hline Mean & 0.01 & 0.01 & 0.01 & 0.01 & 0.01 & 0.03 & 0.02 & 0.02 & 0.02 & 0.05 & 0.12 & 0.14 & 0.18 & 0.06 \\
\hline $\mathrm{s}$ & 0.11 & 0.11 & 0.08 & 0.11 & 0.11 & 0.27 & 0.18 & 0.14 & 0.14 & 0.22 & 0.41 & 0.42 & 0.63 & 0.27 \\
\hline$s^{2}$ & 0.01 & 0.01 & 0.01 & 0.01 & 0.01 & 0.07 & 0.03 & 0.02 & 0.02 & 0.05 & 0.17 & 0.18 & 0.39 & 0.07 \\
\hline $\mathrm{CV}(\%)$ & 874.62 & 874.62 & $1,240.97$ & 874.62 & 874.62 & 819.73 & 922.54 & 711.77 & 711.77 & 428.59 & 351.98 & 293.28 & 357.97 & 419.45 \\
\hline $\mathrm{I}_{\delta}$ & $0.00^{\mathrm{ns}}$ & $0.00^{\mathrm{ns}}$ & - & $0.00^{\mathrm{ns}}$ & $0.00^{\mathrm{ns}}$ & $46.20 *$ & $51.33^{*}$ & $0.00^{\mathrm{ns}}$ & $0.00^{\mathrm{ns}}$ & $0.00^{\mathrm{ns}}$ & $5.03 *$ & $2.67 *$ & $8.34 *$ & $3.42^{\mathrm{ns}}$ \\
\hline \multirow[t]{2}{*}{$\mathrm{k}$} & -1.99 & -1.99 & - & -1.99 & -1.99 & 0.03 & 0.03 & -1.49 & -1.49 & -1.14 & 0.26 & 0.62 & 0.14 & 0.46 \\
\hline & \multicolumn{14}{|c|}{ Euschistus heros } \\
\hline Mínimum & 0.00 & 0.00 & 0.00 & 0.00 & 0.00 & 0.00 & 0.00 & 0.00 & 0.00 & 0.00 & 0.00 & 0.00 & 0.00 & 0.00 \\
\hline Maximum & 0.00 & 0.00 & 0.00 & 0.00 & 1.00 & 2.00 & 1.00 & 1.00 & 1.00 & 1.00 & 1.00 & 1.00 & 2.00 & 3.00 \\
\hline Mean & 0.00 & 0.00 & 0.00 & 0.00 & 0.01 & 0.02 & 0.01 & 0.01 & 0.01 & 0.01 & 0.04 & 0.05 & 0.14 & 0.21 \\
\hline $\mathrm{s}$ & 0.00 & 0.00 & 0.00 & 0.00 & 0.08 & 0.18 & 0.11 & 0.11 & 0.08 & 0.11 & 0.19 & 0.22 & 0.39 & 0.55 \\
\hline$s^{2}$ & 0.00 & 0.00 & 0.00 & 0.00 & 0.01 & 0.03 & 0.01 & 0.01 & 0.01 & 0.01 & 0.04 & 0.05 & 0.15 & 0.30 \\
\hline $\mathrm{CV}(\%)$ & - & - & - & - & $1,240.97$ & 922.54 & 874.62 & 874.62 & $1,240.97$ & 874.62 & 498.28 & 428.59 & 270.56 & 255.68 \\
\hline $\mathrm{I}_{\delta}$ & - & - & - & - & - & $51.33^{*}$ & $0.00^{\mathrm{ns}}$ & $0.00^{\mathrm{ns}}$ & - & $0.00^{\mathrm{ns}}$ & $0.00^{\mathrm{ns}}$ & $0.00^{\mathrm{ns}}$ & $1.33^{\mathrm{ns}}$ & $2.92 *$ \\
\hline \multirow[t]{2}{*}{$\mathrm{k}$} & - & - & - & - & - & 0.03 & -1.99 & -1.99 & - & -1.99 & -1.19 & -1.14 & 3.12 & 0.53 \\
\hline & \multicolumn{14}{|c|}{ Chinavia sp. } \\
\hline Mínimum & 0.00 & 0.00 & 0.00 & 0.00 & 0.00 & 0.00 & 0.00 & 0.00 & 0.00 & 0.00 & 0.00 & 0.00 & 0.00 & 0.00 \\
\hline Maximum & 0.00 & 1.00 & 0.00 & 0.00 & 0.00 & 0.00 & 0.00 & 1.00 & 2.00 & 2.00 & 1.00 & 3.00 & 3.00 & 1.00 \\
\hline Mean & 0.00 & 0.01 & 0.00 & 0.00 & 0.00 & 0.00 & 0.00 & 0.05 & 0.07 & 0.05 & 0.03 & 0.08 & 0.09 & 0.03 \\
\hline $\mathrm{s}$ & 0.00 & 0.11 & 0.00 & 0.00 & 0.00 & 0.00 & 0.00 & 0.21 & 0.28 & 0.25 & 0.16 & 0.36 & 0.42 & 0.16 \\
\hline$s^{2}$ & 0.00 & 0.01 & 0.00 & 0.00 & 0.00 & 0.00 & 0.00 & 0.04 & 0.08 & 0.06 & 0.03 & 0.13 & 0.17 & 0.03 \\
\hline $\mathrm{CV}(\%)$ & - & 874.62 & - & - & - & - & - & 459.75 & 395.56 & 481.80 & 614.37 & 427.25 & 459.75 & 614.37 \\
\hline $\mathrm{I}_{\delta}$ & - & $0.00^{\mathrm{ns}}$ & - & - & - & - & - & $0.00^{\mathrm{ns}}$ & $2.80^{\mathrm{ns}}$ & $5.50 *$ & $0.00^{\mathrm{ns}}$ & $7.90 *$ & $11.85^{*}$ & $0.00^{\mathrm{ns}}$ \\
\hline $\mathrm{k}$ & - & -1.99 & - & - & - & - & - & -1.16 & 0.61 & 0.25 & -1.32 & 0.16 & 0.10 & -1.32 \\
\hline
\end{tabular}

(1) Developmental stages of the soybean according to Ritchie et al. (1982), adapted by Yorinori (1996). * Morisita index different from 1 by the $\chi 2$ test at $5 \% .{ }^{\text {ns }}$ Non-significant. - Not calculated where no stink bugs, or only 1 , were found

Table 3 - Minimum, maximum, mean, standard deviation (s), variance ( $\left.\mathrm{s}^{2}\right)$, coefficient of variation (CV\%), Morisita index $\left(\mathrm{I}_{\delta}\right)$ and $\mathrm{k}$ parameter of the negative binomial distribution for the population density of nymphs, adults, and nymphs and adults of the stink bug, irrespective of species Dichelops furcatus, Piezodorus guildinii, Edessa meditabunda, Nezara viridula, Euschistus heros and Chinavia sp., expressed as stink bugs $\mathrm{m}^{-2}$, based on 154 points each of $1 \mathrm{~m}^{2}$ in area, for 14 phenological stages in the soybean

\begin{tabular}{|c|c|c|c|c|c|c|c|c|c|c|c|c|c|c|}
\hline \multirow{2}{*}{ Statistic } & \multicolumn{14}{|c|}{ - Phenological stage ${ }^{(1)}-{ }_{-}$} \\
\hline & V7 & V9 & V11 & $\mathrm{R} 1$ & $\mathrm{R} 2$ & R3 & $\mathrm{R} 4$ & R5.1 & R5.3 & R5.5 & R6 & R7.1 & R7.3 & R8.2 \\
\hline & \multicolumn{14}{|c|}{ Nymphs } \\
\hline Mínimum & 0.00 & 0.00 & 0.00 & 0.00 & 0.00 & 0.00 & 0.00 & 0.00 & 0.00 & 0.00 & 0.00 & 0.00 & 1.00 & 2.00 \\
\hline Maximum & 0.00 & 1.00 & 4.00 & 5.00 & 4.00 & 5.00 & 4.00 & 6.00 & 5.00 & 40.00 & 28.00 & 34.00 & 54.00 & 44.00 \\
\hline Mean & 0.00 & 0.02 & 0.05 & 0.12 & 0.27 & 0.53 & 0.53 & 0.69 & 0.47 & 1.19 & 3.92 & 9.78 & 14.47 & 10.05 \\
\hline $\mathrm{s}$ & 0.00 & 0.14 & 0.35 & 0.52 & 0.59 & 0.89 & 0.92 & 1.07 & 0.83 & 3.53 & 4.16 & 6.92 & 7.45 & 5.57 \\
\hline$s^{2}$ & 0.00 & 0.02 & 0.12 & 0.27 & 0.35 & 0.80 & 0.84 & 1.15 & 0.70 & 12.47 & 17.27 & 47.86 & 55.54 & 31.00 \\
\hline $\mathrm{CV}(\%)$ & - & 711.77 & 768.75 & 447.70 & 223.32 & 170.05 & 174.17 & 154.65 & 178.31 & 295.57 & 106.12 & 70.74 & 51.51 & 55.39 \\
\hline $\mathrm{I}_{\delta}$ & - & $0.00^{\mathrm{ns}}$ & $44.00^{*}$ & $13.08 *$ & $2.25^{*}$ & $2.00^{*}$ & $2.14 *$ & $1.96^{*}$ & $2.05^{*}$ & $8.89 *$ & $1.87 *$ & $1.40^{*}$ & $1.20^{*}$ & $1.21 *$ \\
\hline $\mathrm{k}$ & - & -1.49 & 0.03 & 0.09 & 0.81 & 1.01 & 0.88 & 1.05 & 0.96 & 0.13 & 1.15 & 2.51 & 5.10 & 4.82 \\
\hline
\end{tabular}


Continuation of Table 3

\begin{tabular}{|c|c|c|c|c|c|c|c|c|c|c|c|c|c|c|}
\hline \multirow[b]{2}{*}{ Mínimum } & \multicolumn{14}{|c|}{ Adults } \\
\hline & 0.00 & 0.00 & 0.00 & 0.00 & 0.00 & 0.00 & 0.00 & 0.00 & 0.00 & 0.00 & 0.00 & 0.00 & 0.00 & 0.00 \\
\hline Maximum & 2.00 & 2.00 & 2.00 & 2.00 & 3.00 & 4.00 & 4.00 & 6.00 & 4.00 & 4.00 & 5.00 & 9.00 & 21.00 & 14.00 \\
\hline Mean & 0.04 & 0.14 & 0.08 & 0.12 & 0.19 & 0.34 & 0.34 & 0.47 & 0.57 & 0.69 & 0.69 & 1.71 & 4.88 & 5.01 \\
\hline $\mathrm{s}$ & 0.25 & 0.36 & 0.32 & 0.35 & 0.54 & 0.68 & 0.70 & 0.86 & 0.85 & 0.89 & 0.94 & 1.79 & 3.68 & 3.07 \\
\hline $\mathrm{s}^{2}$ & 0.06 & 0.13 & 0.10 & 0.12 & 0.29 & 0.46 & 0.49 & 0.73 & 0.72 & 0.79 & 0.88 & 3.21 & 13.55 & 9.41 \\
\hline $\mathrm{CV}(\%)$ & 648.47 & 266.04 & 381.91 & 283.03 & 275.77 & 197.60 & 206.58 & 183.28 & 148.19 & 129.22 & 136.49 & 104.99 & 75.47 & 61.20 \\
\hline $\mathrm{I}_{\delta}$ & $20.53 *$ & $0.73^{\mathrm{ns}}$ & $3.95^{*}$ & $0.90^{\mathrm{ns}}$ & $3.54^{*}$ & $2.01^{*}$ & $2.32^{*}$ & $2.23^{*}$ & $1.45^{*}$ & $1.22^{\mathrm{ns}}$ & $1.41^{*}$ & $1.52^{*}$ & $1.36^{*}$ & $1.17^{*}$ \\
\hline \multirow[t]{2}{*}{$\mathrm{k}$} & 0.06 & -3.91 & 0.37 & -10.55 & 0.40 & 1.00 & 0.77 & 0.82 & 2.24 & 4.61 & 2.44 & 1.94 & 2.74 & 5.71 \\
\hline & \multicolumn{14}{|c|}{ Nymphs + adults } \\
\hline Mínimum & 0.00 & 0.00 & 0.00 & 0.00 & 0.00 & 0.00 & 0.00 & 0.00 & 0.00 & 0.00 & 0.00 & 1.00 & 2.00 & 5.00 \\
\hline Maximum & 2.00 & 2.00 & 4.00 & 5.00 & 5.00 & 7.00 & 6.00 & 9.00 & 6.00 & 40.00 & 28.00 & 35.00 & 56.00 & 51.00 \\
\hline Mean & 0.04 & 0.16 & 0.13 & 0.24 & 0.46 & 0.87 & 0.86 & 1.16 & 1.04 & 1.88 & 4.60 & 11.49 & 19.34 & 15.06 \\
\hline $\mathrm{s}$ & 0.25 & 0.40 & 0.47 & 0.63 & 0.91 & 1.20 & 1.24 & 1.50 & 1.23 & 3.58 & 4.17 & 7.41 & 8.42 & 6.68 \\
\hline $\mathrm{s}^{2}$ & 0.06 & 0.16 & 0.22 & 0.39 & 0.83 & 1.45 & 1.54 & 2.24 & 1.50 & 12.78 & 17.42 & 54.92 & 70.92 & 44.56 \\
\hline $\mathrm{CV}(\%)$ & 648.47 & 255.51 & 359.78 & 260.88 & 197.04 & 138.25 & 143.85 & 128.80 & 117.95 & 189.87 & 90.65 & 64.51 & 43.53 & 44.31 \\
\hline$I_{\delta}$ & $20.53^{*}$ & $1.12^{\mathrm{ns}}$ & $6.48^{*}$ & $3.70^{*}$ & $2.73^{*}$ & $1.76^{*}$ & $1.91^{*}$ & $1.80^{*}$ & $1.43^{*}$ & $4.06^{*}$ & $1.60^{*}$ & $1.33^{*}$ & $1.14^{*}$ & $1.13^{*}$ \\
\hline $\mathrm{k}$ & 0.06 & 8.94 & 0.19 & 0.38 & 0.58 & 1.31 & 1.10 & 1.25 & 2.33 & 0.33 & 1.65 & 3.04 & 7.26 & 7.70 \\
\hline
\end{tabular}

(1) Developmental stages of the soybean according to Ritchie et al. (1982), adapted by Yorinori (1996). * Morisita index different from 1 by the $\chi 2$ test at $5 \%$. ${ }^{\text {ns }}$ Non-significant. - Not calculated where no stink bugs, or only 1 , were found

Chinavia sp. compared to those observed for $P$. guildinii (Tables 1 and 2). These results suggest that to estimate the average population density of $P$. guildinii will require less sample points. In relation to the phase of the bugs, the higher $\mathrm{CV}$ scores for adults suggest the need for a larger sample size compared to that of the nymphs (Tables 1, 2 and 3). These results therefore suggest that sample size varies between the species and stages of the bugs.

The coefficient of variation decreased gradually towards the initial stages (V7 and V9) in relation to the final stages (R7.1, R7.3 and R8.2) (Tables 1, 2 and 3). This behaviour was inversely proportional to that discussed previously for the average population density of stink bugs. These results suggest that to obtain estimates with the same precision, larger sample sizes (number of sampling points) will be needed in the initial stages (larger CVs and lower population densities), with a gradual decrease toward the final stages of the soybean crop (smaller CVs and higher population densities). Moreover, estimates obtained from a single sample size demonstrate less precision in the initial stages and more precision in the final stages.

In practice, the definition of a sample size (number of sampling points) of the desired precision, taken from population densities close to the control level (higher density), is appropriate because it is then the decision is made for the need to control the pest. At lower population densities, it is possible to allow less precision due to the population density being relatively far from the control level, with the risk of damage being thus lower. As soon as the population of stink bugs increased there was a trend towards homogeneity in the area, resulting in a decrease in the coefficient of variation and consequently fewer sampling points being required when sampling. Similar results were obtained by Cargnelutti Filho et al. (2011), i.e. the absence of white grubs at some sampling points (lower population densities) contributed to high coefficients of variation.

The F-test, applied between the variances for population density of stink-bug nymphs and adults, for each species and phenological stage (61 cases), revealed heterogeneous variances $(\mathrm{P} \leq 0.05)$ in 50 cases $(81.97 \%)$ (Table 4). This should be interpreted as demonstrating that in these 50 cases, the sample size for estimating the average population density of the bugs is different for nymphs and adults. Between the variances for the species for each phase and phonological stage, the F-test revealed heterogeneous variances $(\mathrm{P} \leq 0.05)$ in $100 \%$ of the cases, revealing the need to determine the sample size for each species. The variances for the phenological stages, for each stage and species, were heterogeneous $(\mathrm{P} \leq 0.05)$ in 12 cases. In general therefore, the results indicate the need to use the largest variance, having an acceptable error and fixed level of confidence, to determine a single sample size for these species, phases and phonological stages. On the other hand, the existing variability enables the identification of species and phases of bugs and phenological stages with a higher and lower sample size than that required to estimate the average population density. 
Table 4 - Variance of population density of stink-bug nymphs and adults of the species Dichelops furcatus, Piezodorus guildinii, Edessa meditabunda, Nezara viridula, Euschistus heros and Chinavia sp., expressed as stink bugs $\mathrm{m}^{-2}$, based on 154 points each of $1 \mathrm{~m}^{2}$ in area, for 14 phenological stages in the soybean, and values of the F-test for variance homogeneity ( $\mathrm{F}=$ greater variance/smaller variance)

\begin{tabular}{|c|c|c|c|c|c|c|c|c|c|c|c|c|c|c|c|c|}
\hline \multirow{2}{*}{ Species } & \multirow{2}{*}{ Size } & \multicolumn{15}{|c|}{--- } \\
\hline & & V7 & V9 & V11 & R1 & $\mathrm{R} 2$ & R3 & R4 & R5.1 & R5.3 & R5.5 & R6 & R7.1 & R7.3 & $\mathrm{R} 8.2$ & $\mathrm{~F}(4)$ \\
\hline D. furcatus & nymph & - & - & 0.013 & 0.038 & 0.096 & 0.140 & 0.112 & 0.038 & 0.080 & 0.080 & 0.588 & 1.299 & 1.734 & 1.553 & $134.4^{*}$ \\
\hline D. furcatus & adult & 0.006 & 0.085 & 0.087 & 0.038 & 0.083 & 0.122 & 0.220 & 0.140 & 0.241 & 0.249 & 0.063 & 0.314 & 1.230 & 1.288 & $198.3^{*}$ \\
\hline$F^{(2)}$ & & - & - & $6.8 *$ & $1.0^{\mathrm{ns}}$ & $1.2^{\mathrm{ns}}$ & $1.1^{\mathrm{ns}}$ & $2.0^{*}$ & $3.7^{*}$ & $3.0 *$ & $3.1^{*}$ & $9.4^{*}$ & $4.1^{*}$ & $1.4^{*}$ & $1.2^{\mathrm{ns}}$ & \\
\hline P. guildinii & nymph & - & 0.019 & 0.006 & 0.242 & 0.098 & 0.408 & 0.232 & 0.442 & 0.125 & 1.107 & 7.671 & 37.471 & 39.353 & 20.765 & $6,060.4 *$ \\
\hline P. guildinii & adult & 0.032 & 0.019 & 0.013 & 0.068 & 0.166 & 0.193 & 0.107 & 0.232 & 0.291 & 0.391 & 0.425 & 2.112 & 10.335 & 7.534 & $801.0^{*}$ \\
\hline$F^{(2)}$ & & - & $1.0^{\mathrm{ns}}$ & $2.0^{*}$ & $3.5^{*}$ & $1.7^{*}$ & $2.1 *$ & $2.2 *$ & $1.9^{*}$ & $2.3 *$ & $2.8^{*}$ & $18.0^{*}$ & $17.7 *$ & $3.8^{*}$ & $2.8^{*}$ & \\
\hline E. meditabunda & nymph & - & - & - & - & - & 0.077 & 0.256 & 0.151 & 0.044 & 0.159 & 0.311 & 0.511 & 1.551 & 0.356 & $35.5^{*}$ \\
\hline E. meditabunda & adult & - & 0.013 & - & 0.013 & - & 0.019 & 0.032 & 0.119 & 0.067 & 0.143 & 0.189 & 0.138 & 0.224 & 0.193 & $17.4^{*}$ \\
\hline$F^{(2)}$ & & - & - & - & - & - & $4.0^{*}$ & $8.1 *$ & $1.3^{\mathrm{ns}}$ & $1.5^{*}$ & $1.1^{\mathrm{ns}}$ & $1.6^{*}$ & $3.7 *$ & $6.9^{*}$ & $1.8^{*}$ & \\
\hline N. viridula & nymph & - & - & 0.104 & - & 0.067 & 0.061 & 0.051 & 0.149 & 0.044 & 0.169 & 0.215 & 3.729 & 3.229 & 0.789 & $85.4 *$ \\
\hline N. viridula & adult & 0.013 & 0.013 & 0.006 & 0.013 & 0.013 & 0.071 & 0.032 & 0.019 & 0.019 & 0.050 & 0.169 & 0.176 & 0.394 & 0.074 & $60.7 *$ \\
\hline$F^{(2)}$ & & - & - & $16.0 *$ & - & $5.2^{*}$ & $1.2^{\mathrm{ns}}$ & $1.6^{*}$ & $7.7^{*}$ & $2.3^{*}$ & $3.4^{*}$ & $1.3^{\mathrm{ns}}$ & $21.2 *$ & $8.2^{*}$ & $10.6^{*}$ & \\
\hline E. heros & nymph & - & - & - & - & 0.006 & - & 0.013 & 0.039 & 0.013 & 0.026 & 0.445 & 0.119 & 0.044 & 0.182 & $68.6 *$ \\
\hline E. heros & adult & - & - & - & - & 0.006 & 0.032 & 0.013 & 0.013 & 0.006 & 0.013 & 0.038 & 0.050 & 0.149 & 0.300 & $46.2 *$ \\
\hline$F^{(2)}$ & & - & - & - & - & $1.0^{\mathrm{ns}}$ & - & $1.0^{\mathrm{ns}}$ & $3.0^{*}$ & $2.0^{*}$ & $2.0^{*}$ & $11.8^{*}$ & $2.4^{*}$ & $3.4^{*}$ & $1.6^{*}$ & \\
\hline Chinavia sp. & nymph & - & - & - & - & 0.032 & 0.080 & 0.154 & 0.220 & 0.333 & 9.702 & 9.796 & 4.408 & 19.725 & 2.863 & $610.7^{*}$ \\
\hline Chinavia sp. & adult & - & 0.013 & - & - & - & - & - & 0.044 & 0.080 & 0.063 & 0.025 & 0.130 & 0.175 & 0.025 & $13.5 *$ \\
\hline$F^{(2)}$ & & - & - & - & - & - & - & - & $5.0^{*}$ & $4.2^{*}$ & $154.9^{*}$ & $384.7 *$ & $33.9^{*}$ & $112.9^{*}$ & $112.4^{*}$ & \\
\hline D. furcatus & nymph & - & - & 0.013 & 0.038 & 0.096 & 0.140 & 0.112 & 0.038 & 0.080 & 0.080 & 0.588 & 1.299 & 1.734 & 1.553 & $134.4^{*}$ \\
\hline P. guildinii & nymph & - & 0.019 & 0.006 & 0.242 & 0.098 & 0.408 & 0.232 & 0.442 & 0.125 & 1.107 & 7.671 & 37.471 & 39.353 & 20.765 & $6,060.4^{*}$ \\
\hline E. meditabunda & nymph & - & - & - & - & - & 0.077 & 0.256 & 0.151 & 0.044 & 0.159 & 0.311 & 0.511 & 1.551 & 0.356 & $35.5^{*}$ \\
\hline N. viridula & nymph & - & - & 0.104 & - & 0.067 & 0.061 & 0.051 & 0.149 & 0.044 & 0.169 & 0.215 & 3.729 & 3.229 & 0.789 & $85.4 *$ \\
\hline E. heros & nymph & - & - & - & - & 0.006 & - & 0.013 & 0.039 & 0.013 & 0.026 & 0.445 & 0.119 & 0.044 & 0.182 & $68.6^{*}$ \\
\hline Chinavia sp. & nymph & - & - & - & - & 0.032 & 0.080 & 0.154 & 0.220 & 0.333 & 9.702 & 9.796 & 4.408 & 19.725 & 2.863 & $610.7^{*}$ \\
\hline$F^{(3)}$ & & - & - & $16.0^{*}$ & $6.4^{*}$ & $15.2 *$ & $6.7^{*}$ & $19.8^{*}$ & $11.7 *$ & $25.8^{*}$ & $373.5^{*}$ & $45.6^{*}$ & $314.8 *$ & $901.1^{*}$ & $113.9^{*}$ & \\
\hline D. furcatus & adult & 0.006 & 0.085 & 0.087 & 0.038 & 0.083 & 0.122 & 0.220 & 0.140 & 0.241 & 0.249 & 0.063 & 0.314 & 1.230 & 1.288 & $198.3^{*}$ \\
\hline P. guildinii & adult & 0.032 & 0.019 & 0.013 & 0.068 & 0.166 & 0.193 & 0.107 & 0.232 & 0.291 & 0.391 & 0.425 & 2.112 & 10.335 & 7.534 & $801.0^{*}$ \\
\hline E. meditabunda & adult & - & 0.013 & - & 0.013 & - & 0.019 & 0.032 & 0.119 & 0.067 & 0.143 & 0.189 & 0.138 & 0.224 & 0.193 & $17.4^{*}$ \\
\hline N. viridula & adult & 0.013 & 0.013 & 0.006 & 0.013 & 0.013 & 0.071 & 0.032 & 0.019 & 0.019 & 0.050 & 0.169 & 0.176 & 0.394 & 0.074 & $60.7 *$ \\
\hline E. heros & adult & - & - & - & - & 0.006 & 0.032 & 0.013 & 0.013 & 0.006 & 0.013 & 0.038 & 0.050 & 0.149 & 0.300 & $46.2^{*}$ \\
\hline Chinavia sp. & adult & - & 0.013 & - & - & - & - & - & 0.044 & 0.080 & 0.063 & 0.025 & 0.130 & 0.175 & 0.025 & $13.5^{*}$ \\
\hline$F^{(3)}$ & & $5.0^{*}$ & $6.6^{*}$ & $13.4^{*}$ & $5.3^{*}$ & $25.6^{*}$ & $10.1^{*}$ & $17.0^{*}$ & $18.0^{*}$ & $44.8^{*}$ & $30.3^{*}$ & $16.7 *$ & $42.6^{*}$ & $69.2^{*}$ & $295.9^{*}$ & \\
\hline
\end{tabular}

(1) Developmental stages of the soybean according to Ritchie et al. (1982), adapted by Yorinori (1996). (2)* Variances between the phases of stink bug within each combination of species and phenological stage are heterogeneous by one-sided $\mathrm{F}$ test at $5 \%$ probability. ${ }^{\mathrm{ns}}$ homogeneous variances. ${ }^{(3)} *$ Variance between species within each combination of phase and phenological stage are heterogeneous by one-sided F-test at $5 \%$. ${ }^{(4)} *$ Variances between phenological stages within each combination of phase and species are heterogeneous by one-sided F-test at 5\%. - Not calculated where no stink bugs were found

In most cases relating to the population density of stink-bug nymphs and adults of the six bug species, and to the total number of bugs for each phenological stage, the variance was higher than the mean, the Morisita index 
(MORISITA, 1962) was higher than one $(\mathrm{P} \leq 0.05)$, and the $\mathrm{k}$ parameter of the negative binomial distribution tended toward zero $(-10.55 \leq \mathrm{k} \leq 8.94)$ (Tables 1,2 and 3). These results indicate that the stink bugs were distributed as aggregates in the area. When the spatial distribution of insects is aggregate, Cargnelutti Filho et al. (2011) demonstrated that both the expression presented by Karandinos (1976), commonly used for sample-sizing in the area of entomology, and the expression used in this work give the same estimates of sample size, confirming the suitability of both methods.

The sample size (number of sampling points) in estimating the mean $(\mathrm{m})$ of the population density of stink bugs in combinations of phase and bug species and phenological stage, with the semi-amplitude of the confidence interval equal to $10 \%$ of the estimated mean and level of confidence of $95 \%$, ranged from 127 sampling points ( $P$. guildinii, nymph - R8.2 ) and 60,106 sampling points (P. guildinii, nymph - V11, N. viridula, nymph - V11, E. heros, nymph - R2 and R5.5, D. furcatus, adult - V7, N. viridula, adult - V11 and, adult - R2 and R5.3 ) (Tables 5 and 6). In practice, collecting bugs at 60,106 sampling points is difficult. Smaller sample sizes (number of sampling points) have therefore been determined using permitted lower precisions (semiamplitudes of the confidence interval equal to 20,30, 40 and $50 \%$ of the mean). These sample sizes serve as a basis when planning sampling for specific studies to estimate the mean population density of stink-bug nymphs and adults of the species $D$. furcatus, $P$. guildinii, E. meditabunda, N. viridula, E. heros and Chinavia sp. for the phenological stages of the soybean. The number of sampling units is dependent on the degree of accuracy required, which varies with the purpose of the research: population dynamics, crop damage, levels of economic loss and pest control (SILVA; COSTA, 1998).

The maximum acceptable error is debateable, leaving the user to choose the desired precision based on the availability of time and labour. Cargnelutti Filho et al. (2011) observed that for the estimation of a population of white grubs, the sample size for high accuracy was large and difficult to implement. A large sample size increases the time and cost of sampling, whereas smaller sample sizes can result in less precision, which is also undesirable. It is recommended to use sample sizes that result in high precision while saving time and resources.

To estimate the average population of stink bugs with the same precision, fewer sampling points are needed for the species $P$. guildinii in relation to the other five species (Tables 5 and 6). A larger sample size is required for quantification of adult bugs in relation to nymphs (Tables 5, 6 and 7). This difference may be explained by the lower variability of $P$. guildinii and of the nymphs respectively.
The nymphs do not display great mobility in the area, because they are devoid of wings. According Fucarino et al. (2004) the newly-hatched nymphs remain on the egg mass and only after the third instar do they begin their dispersion.

The sample size obtained was higher for the early and intermediate stages of soybean development, and lower for the final stages (R7.1, R7.3 and R8.2). These results therefore confirm previous inferences that there is variability in the sample size (number of sampling points) when estimating the average population density for the phases and species of stink bug and phenological stages. Employing the net method in the soybean, Costa and Link (1980) found that in larger populations of P. guildinii and N. viridula, smaller sizes of sampling unit were defined. Cullen et al. (2000) found that for a greater density of E. heros in the tomato, the sample size was smaller. Lúcio et al. (2009), studying the sample size for mites in the yerba mate, found a variation in the number of samples as a result of the period of evaluation and the degree of pest infestation.

When making a decision about the control of stink bugs in the soybean, it is important to consider the total number of stink bugs. Another aspect to consider is that at population densities close to the control level, quantification should be more accurate, since the wrong estimate at that time can cause a reduction in bean yield. On the other hand, at lower population densities, errors which result in over-estimation are tolerable. Thus, based on the total number of stink bugs (nymphs + adults), the sample size was larger for the initial and intermediate stages of the crop (V7, V9, V11, R1, R2, R3, R4 and R5.1, R5.3 and R5.5), with values ranging between 61 and 1,824 sampling points (error of estimation of $30 \%$ ) (Table 7). In the final stages (R6, R7.1, R7.3 and R8.2), the sample sizes which were calculated were smaller and ranged from 9 to 36 , which may be explained by the higher population densities of the bugs (between 4.60 and 19.34 bugs $\mathrm{m}^{-2}$ ) during this period (Table 3 ). Thirty-six samples are therefore sufficient when estimating the average population density of stink bugs for these 4 phenological stages when there is a greater incidence of bugs .

In general, only a few producers sample a sufficient number of points when quantifying the population of stink bugs in the soybean. Without the correct procedure to determine the density of the infestation, applications of insecticide are made either preventively or at random. This practice has several negative aspects, such as applications not being made at the appropriate time (before the recommended density and control level or with populations that damage the beans) and the increased use of insecticides, with an increase in the cost of production and deleterious effects on natural enemies and other non-target organisms. 
Table 5 - Sample size (number of sampling points) to estimate the average population density of stink-bug nymphs of the species Dichelops furcatus, Piezodorus guildinii, Edessa meditabunda, Nezara viridula, Euschistus heros and Chinavia sp., for errors of estimation equal to 10,20,30, 40 and $50 \%$ of the estimated mean and semi-amplitude of the confidence interval (Error \%), based on 154 points each of $1 \mathrm{~m}^{2}$ in area, for 14 phenological stages

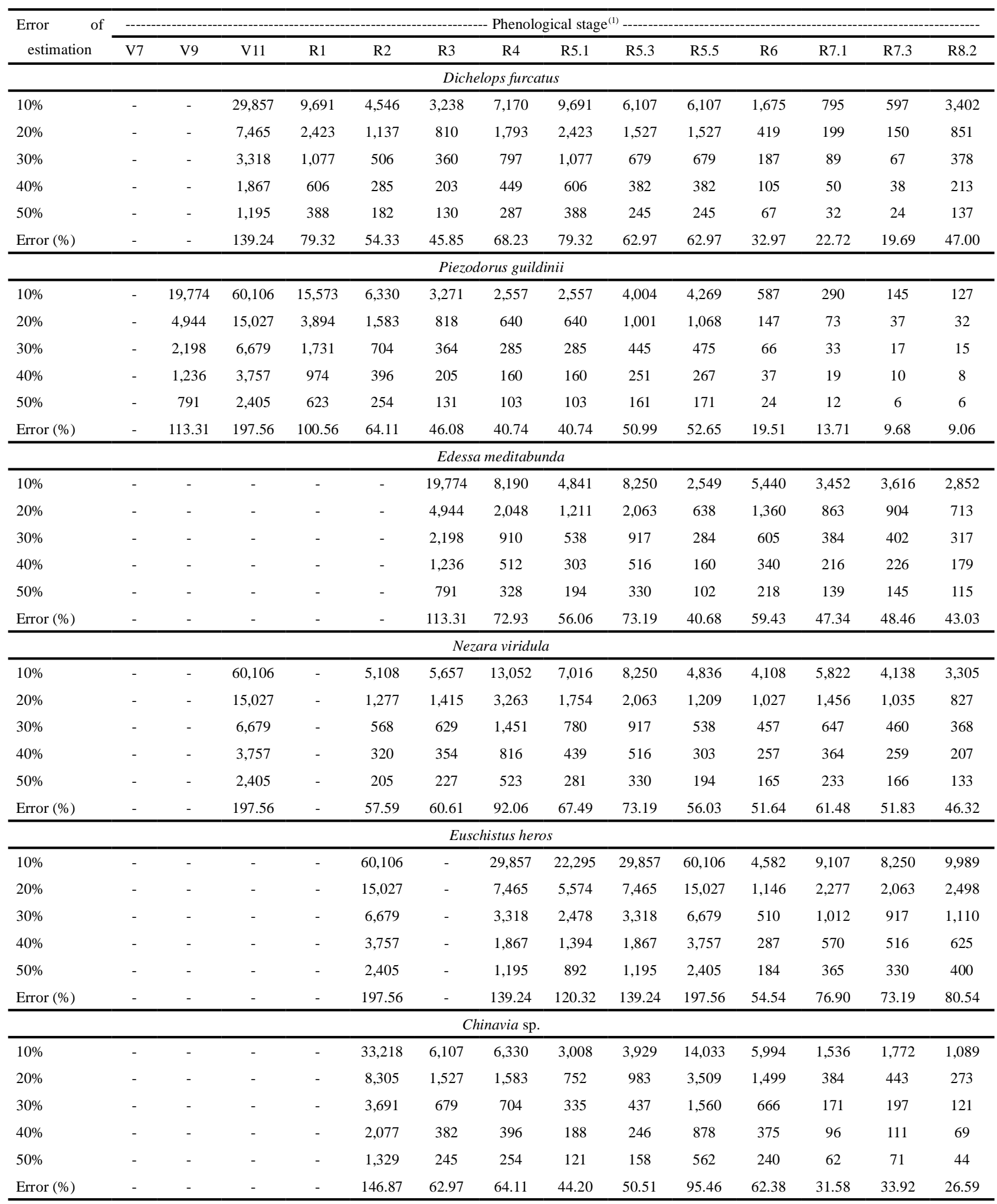

(1) Developmental stages of the soybean according to Ritchie et al. (1982), adapted by Yorinori (1996). - Not calculated where no stink bugs were found 
Table 6 - Sample size (number of sampling points) to estimate the average population density of stink-bug adults of the species Dichelops furcatus, Piezodorus guildinii, Edessa meditabunda, Nezara viridula, Euschistus heros and Chinavia sp., for errors of estimation equal to 10,20,30,40 and $50 \%$ of the estimated mean and semi-amplitude of the confidence interval (Error \%), based on 154 points each of $1 \mathrm{~m}^{2}$ in area, for 14 phenological stages

\begin{tabular}{|c|c|c|c|c|c|c|c|c|c|c|c|c|c|c|}
\hline \multirow{2}{*}{$\begin{array}{l}\text { Error of } \\
\text { estimation }\end{array}$} & \multicolumn{14}{|c|}{ - } \\
\hline & V7 & V9 & V11 & $\mathrm{R} 1$ & $\mathrm{R} 2$ & R3 & R4 & R5.1 & R5.3 & R5.5 & R6 & R7.1 & $\mathrm{R} 7.3$ & $\mathrm{R} 8.2$ \\
\hline \multicolumn{15}{|c|}{ Dichelops furcatus } \\
\hline $10 \%$ & 60,106 & 5,489 & 8,077 & 9,691 & 15,658 & 3,127 & 3,008 & 3,238 & 2,849 & 2,565 & 9,061 & 2,127 & 977 & 968 \\
\hline $20 \%$ & 15,027 & 1,373 & 2,020 & 2,423 & 3,915 & 782 & 752 & 810 & 713 & 642 & 2,266 & 532 & 245 & 242 \\
\hline $30 \%$ & 6,679 & 610 & 898 & 1,077 & 1,740 & 348 & 335 & 360 & 317 & 285 & 1,007 & 237 & 109 & 108 \\
\hline $40 \%$ & 3,757 & 344 & 505 & 606 & 979 & 196 & 188 & 203 & 179 & 161 & 567 & 133 & 62 & 61 \\
\hline $50 \%$ & 2,405 & 220 & 324 & 388 & 627 & 126 & 121 & 130 & 114 & 103 & 363 & 86 & 40 & 39 \\
\hline Error $(\%)$ & 197.56 & 59.70 & 72.42 & 79.32 & 100.83 & 45.06 & 44.20 & 45.85 & 43.01 & 40.81 & 76.70 & 37.16 & 25.18 & 25.06 \\
\hline \multicolumn{15}{|c|}{ Piezodorus guildinii } \\
\hline $10 \%$ & 33,218 & 19,774 & 29,857 & 7,823 & 3,843 & 3,382 & 3,861 & 2,557 & 2,329 & 1,789 & 1,708 & 710 & 313 & 204 \\
\hline $20 \%$ & 8,305 & 4,944 & 7,465 & 1,956 & 961 & 846 & 966 & 640 & 583 & 448 & 427 & 178 & 79 & 51 \\
\hline $30 \%$ & 3,691 & 2,198 & 3,318 & 870 & 427 & 376 & 429 & 285 & 259 & 199 & 190 & 79 & 35 & 23 \\
\hline $40 \%$ & 2,077 & 1,236 & 1,867 & 489 & 241 & 212 & 242 & 160 & 146 & 112 & 107 & 45 & 20 & 13 \\
\hline $50 \%$ & 1,329 & 791 & 1,195 & 313 & 154 & 136 & 155 & 103 & 94 & 72 & 69 & 29 & 13 & 9 \\
\hline Error $(\%)$ & 146.87 & 113.31 & 139.24 & 71.27 & 49.95 & 46.86 & 50.07 & 40.74 & 38.88 & 34.07 & 33.30 & 21.46 & 14.25 & 11.50 \\
\hline \multicolumn{15}{|c|}{ Edessa meditabunda } \\
\hline $10 \%$ & - & 29,857 & - & 29,857 & - & 19,774 & 11,707 & 9,107 & 5,108 & 7,841 & 3,608 & 4,422 & 2,844 & 2,125 \\
\hline $20 \%$ & - & 7,465 & - & 7,465 & - & 4,944 & 2,927 & 2,277 & 1,277 & 1,961 & 902 & 1,106 & 711 & 532 \\
\hline $30 \%$ & - & 3,318 & - & 3,318 & - & 2,198 & 1,301 & 1,012 & 568 & 872 & 401 & 492 & 316 & 237 \\
\hline $40 \%$ & - & 1,867 & - & 1,867 & - & 1,236 & 732 & 570 & 320 & 491 & 226 & 277 & 178 & 133 \\
\hline $50 \%$ & - & 1,195 & - & 1,195 & - & 791 & 469 & 365 & 205 & 314 & 145 & 177 & 114 & 85 \\
\hline Error $(\%)$ & - & 139.24 & - & 139.24 & - & 113.31 & 87.19 & 76.90 & 57.59 & 71.35 & 48.40 & 53.59 & 42.97 & 37.15 \\
\hline \multicolumn{15}{|c|}{ Nezara viridula } \\
\hline $10 \%$ & 29,857 & 29,857 & 60,106 & 29,857 & 29,857 & 26,227 & 33,218 & 19,774 & 19,774 & 7,170 & 4,836 & 3,358 & 5,002 & 6,867 \\
\hline $20 \%$ & 7,465 & 7,465 & 15,027 & 7,465 & 7,465 & 6,557 & 8,305 & 4,944 & 4,944 & 1,793 & 1,209 & 840 & 1,251 & 1,717 \\
\hline $30 \%$ & 3,318 & 3,318 & 6,679 & 3,318 & 3,318 & 2,915 & 3,691 & 2,198 & 2,198 & 797 & 538 & 374 & 556 & 763 \\
\hline $40 \%$ & 1,867 & 1,867 & 3,757 & 1,867 & 1,867 & 1,640 & 2,077 & 1,236 & 1,236 & 449 & 303 & 210 & 313 & 430 \\
\hline $50 \%$ & 1,195 & 1,195 & 2,405 & 1,195 & 1,195 & 1,050 & 1,329 & 791 & 791 & 287 & 194 & 135 & 201 & 275 \\
\hline Error $(\%)$ & 139.24 & 139.24 & 197.56 & 139.24 & 139.24 & 130.50 & 146.87 & 113.31 & 113.31 & 68.23 & 56.03 & 46.69 & 56.99 & 66.78 \\
\hline \multicolumn{15}{|c|}{ Euschistus heros } \\
\hline $10 \%$ & - & - & - & - & 60,106 & 33,218 & 29,857 & 29,857 & 60,106 & 29,857 & 9,691 & 7,170 & 2,858 & 2,552 \\
\hline $20 \%$ & - & - & - & - & 15,027 & 8,305 & 7,465 & 7,465 & 15,027 & 7,465 & 2,423 & 1,793 & 715 & 638 \\
\hline $30 \%$ & - & - & - & - & 6,679 & 3,691 & 3,318 & 3,318 & 6,679 & 3,318 & 1,077 & 797 & 318 & 284 \\
\hline $40 \%$ & - & - & - & - & 3,757 & 2,077 & 1,867 & 1,867 & 3,757 & 1,867 & 606 & 449 & 179 & 160 \\
\hline $50 \%$ & - & - & - & - & 2,405 & 1,329 & 1,195 & 1,195 & 2,405 & 1,195 & 388 & 287 & 115 & 103 \\
\hline Error (\%) & - & - & - & - & 197.56 & 146.87 & 139.24 & 139.24 & 197.56 & 139.24 & 79.32 & 68.23 & 43.07 & 40.70 \\
\hline \multicolumn{15}{|c|}{ Chinavia sp. } \\
\hline $10 \%$ & - & 29,857 & - & - & - & - & - & 8,250 & 6,107 & 9,061 & 14,732 & 7,125 & 8,250 & 14,732 \\
\hline $20 \%$ & - & 7,465 & - & - & - & - & - & 2,063 & 1,527 & 2,266 & 3,683 & 1,782 & 2,063 & 3,683 \\
\hline $30 \%$ & - & 3,318 & - & - & - & - & - & 917 & 679 & 1,007 & 1,637 & 792 & 917 & 1,637 \\
\hline $40 \%$ & - & 1,867 & - & - & - & - & - & 516 & 382 & 567 & 921 & 446 & 516 & 921 \\
\hline $50 \%$ & - & 1,195 & - & - & - & - & - & 330 & 245 & 363 & 590 & 285 & 330 & 590 \\
\hline Error (\%) & - & 139.24 & - & - & - & - & - & 73.19 & 62.97 & 76.70 & 97.81 & 68.02 & 73.19 & 97.81 \\
\hline
\end{tabular}

${ }^{(1)}$ Developmental stages of the soybean according to Ritchie et al. (1982), adapted by Yorinori (1996). - Not calculated where no stink bugs were found 
Table 7 - Sample size (number of sampling points) to estimate the average population density of stink-bug nymphs, adults, and nymphs and adults, irrespective of species Dichelops furcatus, Piezodorus guildinii, Edessa meditabunda, Nezara viridula, Euschistus heros and Chinavia sp., for errors of estimation equal to 10,20,30,40 and 50\% of the estimated mean and semi-amplitude of the confidence interval (Error \%), based on 154 points each of $1 \mathrm{~m}^{2}$ in area, for 14 phenological stages

\begin{tabular}{|c|c|c|c|c|c|c|c|c|c|c|c|c|c|c|}
\hline \multirow{2}{*}{$\begin{array}{l}\text { Error of } \\
\text { estimation }\end{array}$} & \multirow{2}{*}{ V7 } & \multirow[b]{2}{*}{ V9 } & \multirow[b]{2}{*}{ V11 } & \multirow[b]{2}{*}{$\mathrm{R} 1$} & \multirow[b]{2}{*}{$\mathrm{R} 2$} & \multirow[b]{2}{*}{ R3 } & \multirow[b]{2}{*}{$\mathrm{R} 4$} & \multirow{2}{*}{$\mathrm{R} 5.1$} & \multirow[b]{2}{*}{$\mathrm{R} 5.3$} & \multirow[b]{2}{*}{ R5.5 } & \multirow[b]{2}{*}{ R6 } & \multirow[b]{2}{*}{$\mathrm{R} 7.1$} & \multirow[b]{2}{*}{$\mathrm{R} 7.3$} & \multirow{2}{*}{ R8.2 } \\
\hline & & & & & & & & & & & & & & \\
\hline \multicolumn{15}{|c|}{ Nymphs } \\
\hline $10 \%$ & - & 19,774 & 23,066 & 7,823 & 1,947 & 1,129 & 1,184 & 934 & 1,241 & 3,410 & 440 & 196 & 104 & 120 \\
\hline $20 \%$ & - & 4,944 & 5,767 & 1,956 & 487 & 283 & 296 & 234 & 311 & 853 & 110 & 49 & 26 & 30 \\
\hline $30 \%$ & - & 2,198 & 2,563 & 870 & 217 & 126 & 132 & 104 & 138 & 379 & 49 & 22 & 12 & 14 \\
\hline $40 \%$ & - & 1,236 & 1,442 & 489 & 122 & 71 & 74 & 59 & 78 & 214 & 28 & 13 & 7 & 8 \\
\hline $50 \%$ & - & 791 & 923 & 313 & 78 & 46 & 48 & 38 & 50 & 137 & 18 & 8 & 5 & 5 \\
\hline Error (\%) & - & 113.31 & 122.38 & 71.27 & 35.55 & 27.07 & 27.73 & 24.62 & 28.39 & 47.05 & 16.89 & 11.26 & 8.20 & 8.82 \\
\hline \multicolumn{15}{|c|}{ Adults } \\
\hline $10 \%$ & 16,413 & 2,763 & 5,693 & 3,127 & 2,969 & 1,524 & 1,666 & 1,312 & 858 & 652 & 728 & 431 & 223 & 147 \\
\hline $20 \%$ & 4,104 & 691 & 1,424 & 782 & 743 & 381 & 417 & 328 & 215 & 163 & 182 & 108 & 56 & 37 \\
\hline $30 \%$ & 1,824 & 307 & 633 & 348 & 330 & 170 & 186 & 146 & 96 & 73 & 81 & 48 & 25 & 17 \\
\hline $40 \%$ & 1,026 & 173 & 356 & 196 & 186 & 96 & 105 & 82 & 54 & 41 & 46 & 27 & 14 & 10 \\
\hline $50 \%$ & 657 & 111 & 228 & 126 & 119 & 61 & 67 & 53 & 35 & 27 & 30 & 18 & 9 & 6 \\
\hline Error (\%) & 103.23 & 42.35 & 60.80 & 45.06 & 43.90 & 31.46 & 32.89 & 29.18 & 23.59 & 20.57 & 21.73 & 16.71 & 12.02 & 9.74 \\
\hline \multicolumn{15}{|c|}{ Nymphs + Adults } \\
\hline $10 \%$ & 16,413 & 2,549 & 5,053 & 2,657 & 1,516 & 746 & 808 & 648 & 543 & 1,407 & 321 & 163 & 74 & 77 \\
\hline $20 \%$ & 4,104 & 638 & 1,264 & 665 & 379 & 187 & 202 & 162 & 136 & 352 & 81 & 41 & 19 & 20 \\
\hline $30 \%$ & 1,824 & 284 & 562 & 296 & 169 & 83 & 90 & 72 & 61 & 157 & 36 & 19 & 9 & 9 \\
\hline $40 \%$ & 1,026 & 160 & 316 & 167 & 95 & 47 & 51 & 41 & 34 & 88 & 21 & 11 & 5 & 5 \\
\hline $50 \%$ & 657 & 102 & 203 & 107 & 61 & 30 & 33 & 26 & 22 & 57 & 13 & 7 & 3 & 4 \\
\hline Error (\%) & 103.23 & 40.68 & 57.28 & 41.53 & 31.37 & 22.01 & 22.90 & 20.51 & 18.78 & 30.23 & 14.43 & 10.27 & 6.93 & 7.05 \\
\hline
\end{tabular}

${ }^{(1)}$ Developmental stages of the soybean according to Ritchie et al. (1982), adapted by Yorinori (1996). - Not calculated where no stink bugs were found

Any integrated pest management in the soybean requires that planning be based on solid foundations, such as the diagnosis of the species of bug, identification and registering of age throughout the crop cycle, and above all, the quantification of the number of individuals present in the cultivated areas. Thus, a correct, accurate and speedy quantification of the population of pentatomidae in the soybean is the only indicator that can determine the actions necessary for control, and is therefore the way to protect the crop from losses in production, with lesser costs and lower environmental impact.

Knowing the exact number of sampling points in order to obtain the average population density of stink bugs is therefore the first step when planning, followed by carrying out sampling in the field and diagnosis of the population density, which will indicate to producers, technical assistants and administrators the correct time to make the decision about management.

\section{CONCLUSION}

There is variability in the sample size (number of sampling points) when estimating the average population density of stink bugs, between the phases and species of the bugs and between the phenological stages of the soybean. Smaller sample sizes are needed for the nymphs of $P$. guildinii and final phonological stages (R6, R7.1, R7.3 and R8.2) compared to the adults of D. furcatus, E. meditabunda, N. viridula, E. heros and Chinavia sp. and the initial (V7, V9 and V11) and intermediate phenological stages (R1, R2, R3, R4, R5.1, R5.3 and R5.5) of the soybean respectively. Thirty-six sampling sites are sufficient to estimate the average population density of stink bugs in the final phenological stages (R6, R7.1, R7.3 and R8.2) at an error of estimation equal to $30 \%$ of the estimated mean and at a level of confidence of $95 \%$. 


\section{ACKNOWLEDGEMENTS}

The authors wish to thank the National Council for Scientific and Technological Development (CNPq) and the Coordination for the Improvement of Higher Education Personnel (CAPES), for the grant of a scholarship to the authors. Thanks are also due to the scholarship students and volunteers for their assistance in collecting the data.

\section{REFERENCES}

BELORTE, L. C. C; RAMIRO,Z.A.; FARIA,A. M. Levantamento de percevejos pentatomídeos em cinco cultivares de soja [Glycine $\max ($ L.) Merrill, 1917] na região de Araçatuba, SP. Arquivos do Instituto Biológico, v. 70, n. 4, p. 447-451, 2003.

BUSSAB, W. O.; MORETTIN, P. A. Estatística básica. 5. ed. São Paulo: Saraiva, 2004. 526 p.

CARGNELUTTI FILHO, A. et al. Dimensionamento de amostra na estimação da população de corós em áreas de campo nativo e de cultivo no Estado do Rio Grande do Sul. Ciência Rural, v. 41, n. 8, p. 1300-1306, 2011.

COMPANHIA NACIONAL DE ABASTECIMENTO. 2011. Disponível em: <http://www.conab.gov.br/OlalaCMS/uploads/ arquivos/11_11_09_15_03_02_boletim_2o_levantamento_ safra_2011_12.pdf>. Acesso em: 11 abr. 2012.

CORREAA-FERREIRA, B. S. Suscetibilidade da soja a percevejos na fase anterior ao desenvolvimento das vagens. Pesquisa Agropecuária Brasileira, v. 40, n. 11, p. 1067-1072, 2005.

CORRÊA-FERREIRA; B. S.; AZEVEDO, J. de. Soybean seed damage by different species of stink bugs. Agricultural and Forest Entomology, v. 4, n. 2, p. 145-150, 2002.

COSTA, E. C.; LINK, D. Determinação do tamanho da unidade amostral para o método da rede, em soja, para insetos de importância agrícola. Revista do Centro de Ciências Rurais, v. 10, n. 2, p. 115-123, 1980.

CULLEN, E. M. et al. Quantifying trade-offs between pest sampling time and precision in commercial IPM sampling programs. Agricultural Systems, v. 66, n. 2, p. 99-113, 2000.

FUCARINO,A. et al. Chemical and physical signals mediating conspecific and heterospecific aggregation behavior of first instar stink bugs. Journal of Chemical Ecology, v. 30, n. 6, p. 1257-1269, 2004.

GUEDES, J. V. C. et al. Capacidade de coleta de dois métodos de amostragem de insetos-praga da soja em diferentes espaçamentos entre linhas. Ciência Rural, v. 36, n. 4, p. 1299-1302, 2006.
GUEDES, J. V. C. et al. Percevejos da soja: novos cenários, novo manejo. Revista Plantio Direto, v. 12, n. 1, p. 24-30, 2012.

KARANDINOS, M. G. Optimal sample size and comments on some published formulae. Bulletin of the Entomological Society of America, v. 22, n. 4, p. 417-421, 1976.

LOURENÇÃO, A. L. et al. Avaliação de danos de percevejos e de desfolhadores em genótipos de soja de ciclos precoce, semiprecoce e médio. Neotropical Entomology, v. 31, n. 4, p. 623-630, 2002.

LÚCIO, A. D. C. et al. Distribuição espacial e tamanho de amostra para o ácaro-do-bronzeado da erva-mate. Revista Árvore, v. 33, n. 1, p. 143-150, 2009.

MAZIERO, H. et al. Volume de calda e inseticidas no controle de Piezodorus guildinii (Westwood) na cultura da soja. Ciência Rural, v. 39, n. 5, p. 1307-1309, 2009.

MEDEIROS, L.; MEGIER, G. A. Ocorrência e desempenho de Euschistus heros (F.) (Heteroptera: Pentatomidae) em plantas hospedeiras alternativas no Rio Grande do Sul. Neotropical Entomology, v. 38, n. 4, p. 459-463, 2009.

MORISITA, M. Id-index, a measure of dispersion of individuals. Researches on Population Ecology, v. 4, n. 1, p. 01-07, 1962.

RITCHIE, S. W. et al. How a soybean plant develops. Ames: Iowa State University of Science And Technology Cooperative Extension Service, 1982.

EMPRESA BRASILEIRA DE PEQUISA AGROPECUÁRIA. Indicações técnicas para a cultura da soja no Rio Grande do Sul e em Santa Catarina 2010/2011 e 2011/2012. Cruz Alta: Fundacep Fecotrigo, 2010. 168 p.

SANTOS, R. S. S. dos. Levantamento populacional de percevejos e da incidência de parasitóides de ovos em cultivos orgânicos de soja. Pesquisa Agropecuária Gaúcha, v. 14, n. 1, p. $41-46,2008$.

SILVA, M. T. B. da et al. Erro e resistência. Revista Cultivar: Grandes Culturas, v. 9, n. 82, p. 22-25, 2007.

SILVA, M. T. B.; COSTA, E. C. Tamanho e número de unidades de amostra de solo para amostragem de larvas de Diloboderus abderus (Sturm) (Coleoptera: Melolonthidae) em plantio direto. Anais da Sociedade Entomológica do Brasil, v. 27, n. 2, p. 193-197, 1998.

STÜRMER, G. R. et al. Eficiência de métodos de amostragem de lagartas e de percevejos na cultura de soja. Ciência Rural, v. 42, n. 12, p. 2105-2111, 2012.

YORINORI, J. T. Cancro da haste da soja: epidemiologia e controle. Londrina: Embrapa Soja, 1996, 75 p. (Circular técnica, 14). 